\title{
Association of TREM-1, IL-1 $\beta$, IL-33/ST2, and TLR Expressions With the Pathogenesis of Ocular Toxoplasmosis in Mouse Models on Different Genetic Backgrounds
}

\section{OPEN ACCESS}

Edited by:

Lihua Xiao,

South China Agricultural University,

China

Reviewed by:

Vera Lucia Pereira-Chioccola,

Instituto Adolfo Lutz, Brazil

Longxian Zhang,

Henan Agricultural University, China

*Correspondence:

Shiguang Huang

thshg@126.com

Fangli LU

fanglilu@yahoo.com

Specialty section:

This article was submitted to

Infectious Diseases,

a section of the journal

Frontiers in Microbiology

Received: 24 April 2019

Accepted: 17 September 2019

Published: 09 October 2019

Citation:

Zhang $Y$, He J, Zheng $H$, Huang S and Lu F (2019) Association of TREM-1, IL-1 $\beta, I L-33 / S T 2$, and TLR Expressions With the Pathogenesis of Ocular Toxoplasmosis in Mouse Models on Different Genetic Backgrounds. Front. Microbiol. 10:2264 doi: 10.3389/fmicb.2019.02264

\author{
Yanxia Zhang ${ }^{1,2}$, Jian He ${ }^{1,2,3}$, Huanqin Zheng ${ }^{1,2}$, Shiguang Huang ${ }^{4 *}$ and Fangli Lu ${ }^{1,2 *}$ \\ ${ }^{1}$ Department of Parasitology, Zhongshan School of Medicine, Sun Yat-sen University, Guangzhou, China, ${ }^{2}$ Key Laboratory \\ of Tropical Disease Control of Ministry of Education, Sun Yat-sen University, Guangzhou, China, ${ }^{3}$ Public Experimental \\ Teaching Center, Sun Yat-sen University, Guangzhou, China, ${ }^{4}$ School of Stomatology, Jinan University, Guangzhou, China
}

Ocular toxoplasmosis (OT) is one of the most common causes of posterior uveitis. The signaling of triggering receptor expressed on myeloid cells (TREM)- 1 amplifies inflammation, whereas TREM-2 signaling is anti-inflammatory. IL-1 $\beta$ is a major driver of inflammation during infection. Toll-like receptors (TLRs) play important roles in protective immune response during Toxoplasma gondii infection, and interleukin (IL)-33 receptor (T1/ST2) signaling prevents toxoplasmic encephalitis in mice. However, the pathogenic mechanisms of OT are not yet well elucidated. To investigate the role of TREM-1, TREM-2, IL-1 $\beta$, IL-33/ST2, and TLRs in OT of susceptible C57BL/6 (B6) and resistant BALB/c mice, both strains of mice were intravitreally infected with 500 tachyzoites of the $\mathrm{RH}$ strain of T. gondii. Histopathological analysis showed that T. gondii-infected B6 mice had more severe ocular damage observed by light microscopy, higher number of neutrophil elastase-positive cells in the eyes detected by immunohistochemical staining, more T. gondii tachyzoites in the eyes observed by transmission electron microscopy, and higher mRNA expression levels of tachyzoite-specific surface antigen 1 detected by quantitative real-time reverse transcription-polymerase chain reaction (qRT-PCR) in comparison of $T$. gondii-infected BALB/c mice. Detected by using qRT-PCR, the mRNA expression levels of TREM-1, IL-1 $\beta$, IL-33, ST2, TLR11, TLR12, and TLR13 were significantly higher in the eyes of $T$. gondii-infected B6 mice than those of $T$. gondiiinfected BALB/c mice, whereas the mRNA expression levels of TLR3 and TLR9 were significantly higher in the eyes of $T$. gondii-infected BALB/c mice than those of $T$. gondiiinfected B6 mice. Correlation analysis showed that significant positive correlations existed between TREM-1 and IL-1//L-33/ST2/TLR9/TLR11 in the eyes of B6 mice and existed between TREM-1 and IL-33/ST2/TLR3/TLR9/TLR13 in the eyes of BALB/c mice after ocular T. gondii infection. Our data revealed that, compared with $T$. gondii-resistant BALB/c mice, ocular T. gondii infection can stimulate higher production of TREM-1, IL-33, ST2, TLR11, TLR12, and TLR13 in the eyes of T. gondii-susceptible B6 mice, however, whether those lead to more severe ocular pathology in the susceptible B6 mice remain to be further studied.

Keywords: ocular toxoplasmosis, C57BL/6 mice, BALB/c mice, TREM-1, IL-1 $\beta$, IL-33/ST2, TLRs, neutrophils 


\section{INTRODUCTION}

Ocular toxoplasmosis (OT) is caused by Toxoplasma gondii infection, with potentially vision-threatening complications such as retinal detachment, choroidal neovascularization, and glaucoma (Park and Nam, 2013; Garweg, 2016), which involves typically the posterior part of eye but results in different clinical symptoms based on the involved area and level of inflammation (Ali-Heydari et al., 2013; Laboudi and Sadak, 2017). Eye injuries caused by T. gondii infection affect the retina and the choroid with local inflammatory reactions (Maenz et al., 2014). Acquired infections may account for a larger portion of OT than congenital toxoplasmosis (Atmaca et al., 2004).

It has been reported that genetic factors are major determinants for susceptibility to infection with $T$. gondii (Deckert-Schluter et al., 1994). Our previous study found that compared with both $\mathrm{BALB} / \mathrm{c}$ and $\mathrm{CBA} / \mathrm{J}$ mice, ocular infection of C57BL/6 (B6) mice with T. gondii resulted in severe inflammatory lesions and high numbers of parasites in eye tissue, and higher serum levels of gamma interferon and tumor necrosis factor alpha $(\mathrm{TNF} \alpha)$, indicating that genetic factors of the host are critical in determining susceptibility to experimental OT in murine models ( $\mathrm{Lu}$ et al., 2005). Our recent study demonstrated that B6 mice expressed higher levels of Gal-9 and its receptors (Tim-3 and CD137) in the eye tissues than those in $\mathrm{BALB} / \mathrm{c}$ mice following ocular T. gondii infection (Chen et al., 2017). However, so far genetic factors in the pathogenesis and course of OT still remain unclear.

The triggering receptor expressed on myeloid cells (TREM) family including TREM-1, TREM-2, TREM-3, and TREM-4 have been identified, in which TREM1 activation amplifies inflammation, whereas TREM-2 activation is anti-inflammatory (Klesney-Tait et al., 2006; Turnbull et al., 2006; Watarai et al., 2008). TREM-1 can trigger the release of proinflammatory cytokines such as interleukin (IL)-1 $\beta$, IL-6, and TNF $\alpha$; crucially amplify both acute inflammatory responses and chronic inflammation (Bouchon et al., 2000; Bleharski et al., 2003; Schenk et al., 2007). TREM-1 works synergistically with toll-like receptors (TLRs) and Nod-like receptors to increase proinflammatory reactions (Bouchon et al., 2001; Netea et al., 2006). In addition, TREM-1 plays critical roles in fungal keratitis and increases with growing keratomycosis severity ( $\mathrm{Hu}$ et al., 2014). TREM-2 promotes host resistance to Pseudomonas aeruginosa infection by suppressing corneal inflammation (Sun et al., 2013).

IL-1 $\beta$ is an important inflammation mediator and a proinflammatory cytokine (Zhang et al., 2018), which is involved in multiple cellular activities such as cell proliferation, apoptosis, and differentiation (Zhao et al., 2018). IL-33 is a member of the IL-1 family and has been identified as a mediator of various inflammatory diseases such as asthma, cardiovascular diseases, and allergic diseases (Liew et al., 2016). ST2 is defined as the IL-33 receptor (Carriere et al., 2007). It has been reported that ST2/IL-33 signaling implicates in protection from various infections (Griesenauer and Paczesny, 2017). When IL-33 receptor (T1/ST2)-deficient $\mathrm{BALB} / \mathrm{c}$ mice were infected with T. gondii, they showed increased pathology and increased parasite transcript levels in the brain, indicating T1/ST2 signaling is necessary to prevent the development of toxoplasmic encephalitis (Jones et al., 2010).

Toll-like receptors play an important role in initiating immune responses against many pathogens, including $T$. gondii. TLR/MyD88 signaling pathway is the key pathway in initiating defense against T. gondii (Denkers, 2010). TLR2 and TLR4 contribute to the recognition and stimulation of immunity to $T$. gondii and participate in the host protection to T. gondii infection (Mukherjee et al., 2016). TLR3 induces type I interferon responses via parasite RNA (Beiting et al., 2014). TLR4 and TLR9 single nucleotide polymorphisms are involved in protection against congenital toxoplasmosis (Wujcicka et al., 2015). Mice lacking TLR9 significantly reduce intestinal pathology, lose weight, and live longer than wild-type mice (Minns et al., 2006). A study has proved a role for TLR9 in initiating proinflammatory responses that cause severe OT in Brazil (Peixoto-Rangel et al., 2009). TLR11 can interact with T. gondii profilin-like protein to elicit immune responses (Hatai et al., 2016). TLR12 can function alone in plasmacytoid dendritic cells and interact with TLR11 to specifically recognize and respond to T. gondii profilin (Koblansky et al., 2013). Quadruple TLR3/TLR7/TLR9/TLR11 deficient mice showed diminished resistance to $T$. gondii infection, indicating they play an essential role in toxoplasmosis (Andrade et al., 2013). So far, the role of TREMs and TLRs in OT remains poorly understood. Therefore, this study intended to investigate the expression and role of TREM-1, TREM-2, IL-1 $\beta$, IL33, ST2, and TLRs in OT in mouse models on different genetic backgrounds.

\section{MATERIALS AND METHODS}

\section{Mice, T. gondii Parasites, and Intravitreal Infection}

Female B6 and BALB/c mice, 8-10 weeks old, were purchased from the animal facility at Sun Yat-sen University in Guangzhou, China. T. gondii RH strain tachyzoites were maintained in Vero or human foreskin fibroblast cells grown in Dulbecco's modified Eagle medium (Invitrogen, Carlsbad, CA, United States) supplemented with $5 \%$ fetal bovine serum at $37^{\circ} \mathrm{C}$ with $5 \% \mathrm{CO}_{2}$.

Mice were injected into the mid-vitreous with $1 \mu \mathrm{L}$ parasite suspension in sterile phosphate-buffered saline (PBS, pH 7.4) containing 500 tachyzoites or the same volume of PBS using a 10- $\mu$ L Hamilton microsyringe (Charles et al., 2007). A total of 24 mice of each strain were used in the experiments: 12 mice of each strain were intraocularly injected with 500 tachyzoites of T. gondii and 12 mice of each strain were injected with equal volume of PBS as negative controls. Mice were euthanized at day 8 post infection (p.i.), and the eyes were enucleated for further analysis. 


\section{mRNA Expression Analysis by Using Quantitative Real-Time Reverse Transcription-Polymerase Chain Reaction (qRT-PCR)}

Total RNA was extracted from the eyes of each mouse using a RNA Extraction Kit (TaKaRa Bio Inc., Shiga, Japan) according to the manufacturer's protocol. The absorbance values at 260 and $280 \mathrm{~nm}$ were used to estimate total RNA purity (NanoDrop Technologies, DE, United States). For cDNA synthesis, a PrimeScript ${ }^{\mathrm{TM}}$ II 1st Strand cDNA Synthesis Kit (TaKaRa Bio Inc.) was used; total amount of RNA used in qRT-PCR for each sample was $1 \mu \mathrm{g}$. To determine tissue mRNA levels of TREM-1, TREM-2, IL-1 $\beta$, IL-33, ST2, TLR2, TLR3, TLR4, TLR5, TLR7, TLR9, TLR11, TLR12, and TLR13, qRT-PCR was performed using SYBR Green qPCR Master Mix (TaKaRa Bio Inc.) following the manufacturer's instructions. For eye parasite burden, mRNA level of tachyzoite-specific surface antigen 1 (SAG1) was measured by qRT-PCR as previously described (Liu et al., 2018). All qRT-PCR reactions were performed in duplicate. Primers used for qRT-PCR are listed in Table 1. The results are expressed as relative mRNA levels whereby the expression in naive mice was arbitrarily set at 1 (van der Kraan et al., 2001). Relative mRNA expressions of each target gene were normalized to that of the housekeeping gene, $\beta$-actin, and the results are expressed as fold change compared with uninfected controls.

\section{Histopathology}

Mice were euthanized at day 8 p.i., and the eyes were harvested, immediately fixed in $10 \%$ neutral buffered formalin for $24 \mathrm{~h}$, then transferred to $70 \%$ ethanol, embedded in paraffin. Fourmicrometer-thick sections of the eyes from each mouse were cut, processed through graded alcohols. The sections were denitrified by xylene and rehydrated with graded alcohols (100-70\%), and stained with hematoxylin and eosin (H\&E) (Sigma-Aldrich, St. Louis, MO, United States).

\section{Immunohistochemical Staining for Neutrophils in the Eyes}

For immunohistochemical purposes, after the sections $(4-\mu \mathrm{m})$ were deparaffinized and rehydrated in distilled water. Heatinduced antigen retrieval was performed in citrate buffer in an $800-\mathrm{W}$ microwave oven for $30 \mathrm{~min}$. Sections were treated

TABLE 1 | Primer sequences of mouse target cytokines and housekeeping gene used for quantitative real-time polymerase chain reaction assays.

\begin{tabular}{|c|c|c|}
\hline Genes & Primer sequence $\left(5^{\prime} \rightarrow 3^{\prime}\right)$ & Accession \\
\hline \multirow[t]{2}{*}{ SAG1 } & Forward primer ATGTCGCTTCTTAGCCGAGT & XM_002365028.1 \\
\hline & Reverse primer TCACAGGAAGTTGCTTCAGG & \\
\hline \multirow[t]{2}{*}{$\beta$-actin } & Forward primer TGGAATCCTGTGGCATCCATGAAAC & NM_007393.5 \\
\hline & Reverse primer TAAAACGCAGCTCAGTAACAGTCCG & \\
\hline \multirow[t]{2}{*}{ TLR2 } & Forward primer GCAAACGCTGTTCTGCTCAG & NM_011905.3 \\
\hline & Reverse primer AGGCGTCTCССTСTATTGTATT & \\
\hline \multirow[t]{2}{*}{ TLR3 } & Forward primer GTGAGATACAACGTAGCTGACTG & NM_126166.5 \\
\hline & Reverse primer TCCTGCATCCAAGATAGCAAGT & \\
\hline \multirow[t]{2}{*}{ TLR4 } & Forward primer ATGGCATGGCTTACACCACC & NM_021297.3 \\
\hline & Reverse primer GAGGCCAATITTGTCTCCACA & \\
\hline \multirow[t]{2}{*}{ TLR5 } & Forward primer TGGGGACCCAGTATGCTAACT & NM_016928.3 \\
\hline & Reverse primer CCACAGGAAAACAGCCGAAGT & \\
\hline \multirow[t]{2}{*}{ TLR7 } & Forward primer ATGTGGACACGGAAGAGACAA & NM_133211.4 \\
\hline & Reverse primer ACCATCGAAACCCAAAGACTC & \\
\hline \multirow[t]{2}{*}{ TLR9 } & Forward primer ATGGTTCTCCGTCGAAGGACT & NM_031178.2 \\
\hline & Reverse primer GAGGCTTCAGCTCACAGGG & \\
\hline \multirow[t]{2}{*}{ TLR11 } & Forward primer TCCCTGATTGCATCATAGCAGA & NM_205819.3 \\
\hline & Reverse primer GGGCCGAGGTACAGAATGG & \\
\hline \multirow[t]{2}{*}{ TLR12 } & Forward primer CCTGGTCTCCCGCTATITCAC & NM_205823.2 \\
\hline & Reverse primer CCGAGGTACAACTTCCAAGGT & \\
\hline \multirow[t]{2}{*}{ TLR13 } & Forward primer GTTGTAACCTGGATGCCTAAGAC & NM_205820.1 \\
\hline & Reverse primer GGCCTCTGTCAAGTTGGTGA & \\
\hline \multirow[t]{2}{*}{ TREM-1 } & Forward primer CCTGTTGTGCTCTTCCATCCTG & NC_000083.6 \\
\hline & Reverse primer CGGGTTGTAGTTGTGTCACTGG & \\
\hline \multirow[t]{2}{*}{ TREM-2 } & Forward primer CTACCAGTGTCAGAGTCTCCGA & NC_000083.6 \\
\hline & Reverse primer CCTCGAAACTCGATGACTCCTC & \\
\hline \multirow[t]{2}{*}{ ST2 } & Forward primer CAAGTAGGACCTGTGTGCCC & NC_000067.6 \\
\hline & Reverse primer CGTGTCCAACAATTGACCTG & \\
\hline \multirow[t]{2}{*}{ IL-33 } & Forward primer TCCAACTCCAAGATITCCCCG & NC_000085.6 \\
\hline & Reverse primer CATGCAGTAGACATGGCAGAA & \\
\hline \multirow[t]{2}{*}{$\| \mathrm{L}-1 \beta$} & Forward primer CGCAGCAGCACATCAACAAGAGC & NC_000068.7 \\
\hline & Reverse primer TGTCCTCATCCTGGAAGGTCCACG & \\
\hline
\end{tabular}


with $3 \%$ hydrogen peroxide in methanol for $10 \mathrm{~min}$ at room temperature to inactivate endogenous peroxidase, and then incubated in 5\% normal goat serum in PBS ( $\mathrm{pH} 7.4$ ) for $60 \mathrm{~min}$ at room temperature to block non-specific binding. After washing with PBS, sections were incubated with polyclonal antielastase (1:100 dilutions) (Boster Biological Technology, Wuhan, China) overnight at $4^{\circ} \mathrm{C}$. Negative controls were performed without a primary antibody. Slides were rinsed three times with PBS and were then incubated with the secondary antibody (Goat anti-rabbit IgG) conjugated with horseradish peroxidase for $20 \mathrm{~min}$. The color reaction was revealed by reacting the specimen with a 3,3'-diaminobenzidine substrate (Zhongshan Golden Bridge Technology, Beijing, China). Sections used as isotype controls were incubated with secondary antibodies alone. The sections were counterstained with hematoxylin and positive cells were identified by dark-brown staining under a light microscope.

\section{Transmission Electron Microscopy}

Mice were euthanized at day 8 p.i., and the eyes were dissected, excising the cornea, iris, lens, and vitreous body, and cut into four pieces. The samples were immediately fixed in 3\% glutaraldehyde and $1 \%$ osmium tetroxide (both in $100 \mathrm{mM}$ PBS, $\mathrm{pH}$ 7.2) overnight before being dehydrated through a series of graded ethanol solutions. The fixed tissues were then embedded in SPIPon 812 Embedding Kit (Structure Probe Inc., West Chester, PA, United States) following the manufacture's instruction. Ultrathin sections $(70 \mathrm{~nm})$ were cut from the embedded tissues using the Leica EM UC6 ultramicrotome (Leica Microsystems, Wetzlar, Germany) and mounted on formvar-coated grids. The sections were then stained for $15 \mathrm{~min}$ in aqueous $1 \%$ uranyl acetate followed by $0.2 \%$ lead citrate, and were then analyzed under a JEM100CX-II transmission electron microscope (JEOL Ltd., Tokyo, Japan) at an accelerating voltage of $100 \mathrm{kV}$.

\section{Statistical Analysis}

Statistical analysis was performed using IBM SPSS Statistics version 22.0 (IBM Corp., Armonk, NY, United States). All graphs were generated using GraphPad Prism 7 software (GraphPad software). Data are presented as mean \pm standard deviation (SD) at least three independent biological replicates. Student's $t$ test was used to ascertain the differences between groups. Pearson correlation testing was used for the associations between the levels of cytokines. A value of $P<0.05$ was considered significant.

\section{RESULTS}

\section{Ocular Pathology and Parasite Burden in the Eyes of B6 and BALB/c Mice Infected With T. gondii}

Histopathological studies showed that the intravitreal inoculation of tachyzoites of T. gondii in both B6 and BALB/c mice caused a strong influx of inflammatory cells, thereby destroying and disrupting the normal structures of retina and choroid as compared with the control groups. In contrast with $\mathrm{BALB} / \mathrm{c}$ mice,
B6 mice showed great multiplication of T. gondii tachyzoites with considerable infiltration of numerous migrating cells in the eye tissues at day 8 p.i. (Figure 1A). As for the parasite burden in the eyes with T. gondii infection, there was significantly increased mRNA expression level of $T$. gondii tachyzoite SAG1 gene in the eyes of B6 mice than that in BALB/c mice $(P<0.01)$ (Figure $1 B$ ). The ultrastructural characterization of $T$. gondii tachyzoites in the eyes of B6 and BALB/C mice were captured by means of transmission electron microscopy. More T. gondii tachyzoites were observed in the eyes of B6 mice than those in BALB/C mice (Figure 2).

\section{Neutrophils in the Eyes of T. gondii-Infected B6 and BALB/C Mice}

Neutrophils, a kind of inflammatory cell, were observed in the eyes of T. gondii-infected mice. Neutrophil elastase is a cytotoxic serine protease, which is stored in the azurophilic granules of neutrophil granulocytes and is released by activated neutrophils. Elastase-positive neutrophils were observed in the destroyed retina and choroid, especially evident in the choroid area of both T. gondii-infected B6 and BALB/c mice at day 8 p.i., while they were not observed in those of uninfected B6 and BALB/c mice (Figure 3A). The quantitative analysis showed that compared with T. gondii-infected BALB/c mice, the number of neutrophils was significantly higher in the eyes of T. gondii-infected B6 mice $(P<0.001)$ (Figure 3B).

\section{Expressions of TREM-1, TREM-2, IL-1 $\beta$, IL-33, and ST2 Genes in the Eyes of T. gondii-Infected B6 and BALB/C Mice}

Compared with naive mice, the mRNA expression levels of TREM-1 $(P<0.01$ and $P<0.001$, respectively), IL-33 $(P<0.01)$, and ST2 $(P<0.001)$ were significantly increased in the eyes of both $T$. gondii-infected B6 and BALB/c mice, while IL-1 $\beta$ expression level was significantly increased in that of T. gondii-infected B6 mice at day 8 p.i. $(P<0.001)$. There was insignificant difference in TREM-2 expression levels between T. gondii-infected mice and naive mice. Compared with T. gondiiinfected BALB/c mice, the levels of TREM-1 $(P<0.001)$, IL-1 $\beta(P<0.001)$, IL-33 $(P<0.01)$, and ST2 $(P<0.001)$ were significantly increased in the eyes of T. gondii-infected B6 mice (Figure 4).

\section{Expressions of TLR Genes in the Eyes of T. gondii-Infected B6 and BALB/c Mice}

Compared with uninfected control mice, the expression levels of TLR2, TLR4, TLR5, TLR7, TLR9, TLR11, TLR12, and TLR13 in the eyes of both T. gondii-infected B6 and BALB/c mice were significantly increased at day 8 p.i. (Figure 5). Compared with T. gondii-infected BALB/c mice, the levels of TLR9 $(P<0.001)$, TLR11 $(P<0.001)$, TLR12 $(P<0.001)$, and TLR13 $(P<0.01)$ were significantly increased in the eyes of T. gondii-infected B6 mice at day 8 p.i. However, there were significantly elevated levels of TLR3 and TLR9 in the eyes of T. gondii-infected BALB/c mice $(P<0.05)$ compared with T. gondii-infected B6 mice. 


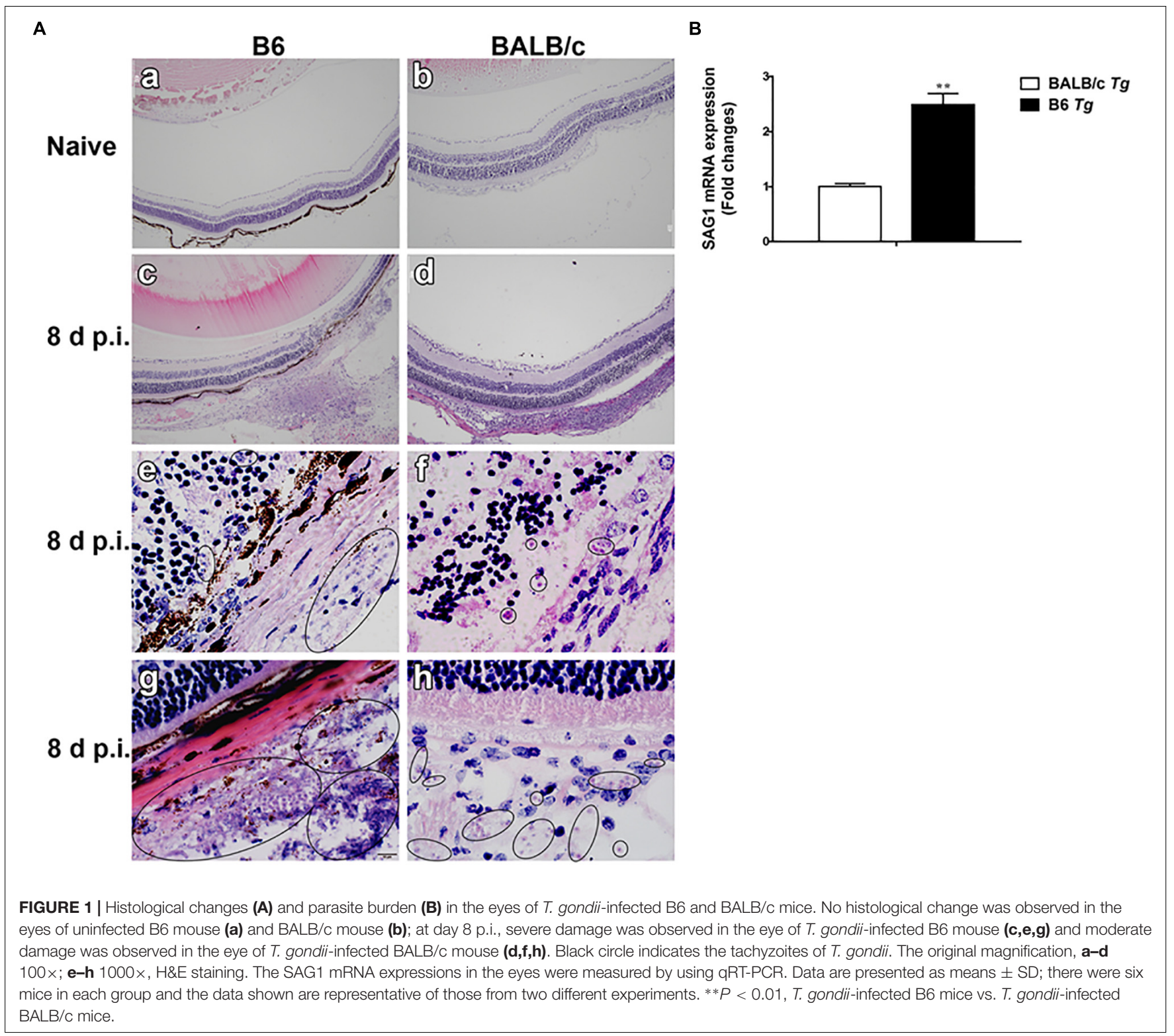

\section{Correlations Between TREM-1 and IL-1 $\beta$, IL-33, ST2, or TLRs in the Eyes of T. gondii-Infected B6 and BALB/c Mice}

The correlations between mRNA levels of TREM- 1 and IL-1 $\beta /$ IL33/ST2/TLRs in the eyes of T. gondii-infected B6 and BALB/c mice were analyzed. Only significant correlations were shown. In T. gondii-infected B6 mice, there were significant correlations between the mRNA levels of TREM-1 and IL-1 $\beta(r=0.8913$, $P=0.0014)$, TREM-1 and IL-33 $(r=0.8534, P=0.0030)$, TREM- 1 and ST2 $(r=0.8371, P=0.0039)$, TREM- 1 and TLR9 $(r=0.7263, P=0.0149)$, and TREM-1 and TLR11 $(r=0.8261$, $P=0.0046)$. In $T$. gondii-infected $\mathrm{BALB} / \mathrm{c}$ mice, there were significant correlations between the mRNA levels of TREM- 1 and TLR3 $(r=0.6610, P=0.0262)$, TREM-1 and IL-33 $(r=0.8751$, $P=0.0020)$, TREM-1 and ST2 $(r=0.7642, P=0.0101)$, TREM- 1 and TLR9 $(r=0.8389, P=0.0038)$, and TREM-1 and TLR13 $(r=0.8019, P=0.0064)$ (Figure 6).

\section{DISCUSSION}

Our previous study has demonstrated that genetic factors of both mouse strains and parasite strains are crucial in determining susceptibility to experimental murine OT (Lu et al., 2005). IL$10, \mathrm{CD}^{+}, \mathrm{CD}^{+} \mathrm{T}$ cells, and $\mathrm{B}$ cells play important roles during murine OT ( $\mathrm{Lu}$ et al., 2003, 2004). Ocular infection caused by $T$. gondii may result in inflammation in the retina, choroid, and uvea (Jasper et al., 2017). In this study, after ocular T. gondii infection, there were more severe ocular pathology, more tachyzoites, and higher parasite load in the eyes of B6 mice than those in $\mathrm{BALB} / \mathrm{c}$ mice. Immunohistochemical staining 

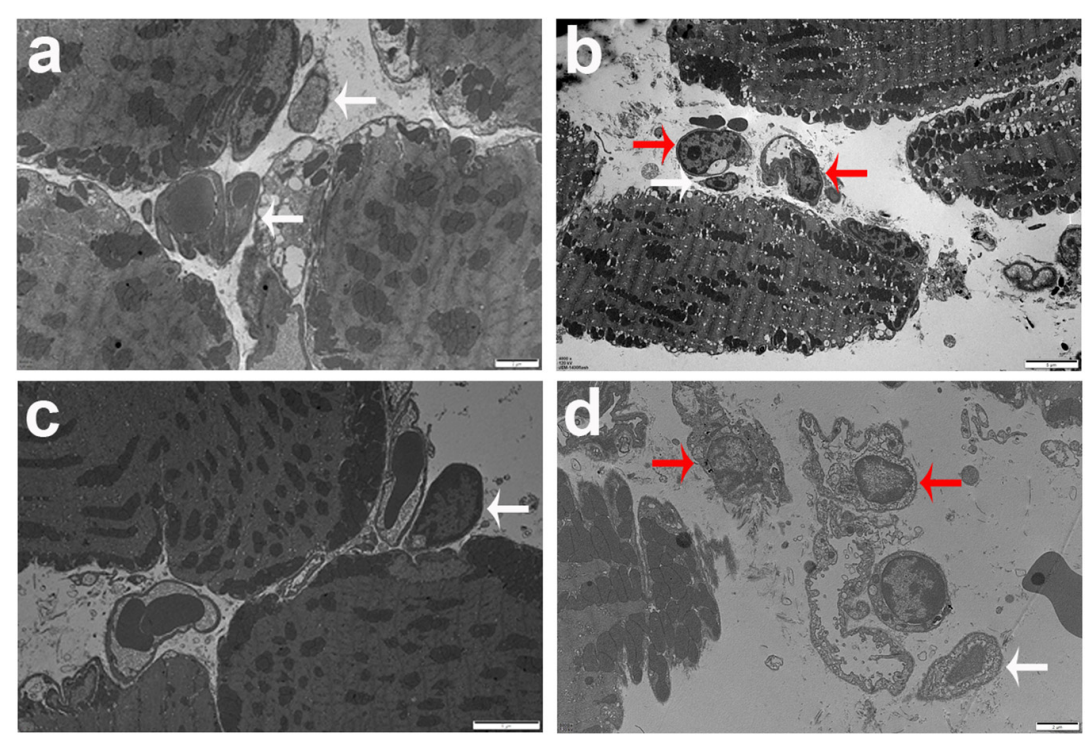

FIGURE 2 | Ultrastructural analysis of eye tissues after T. gondii infection. The images of transmission electron microscopy showed T. gondii in the eyes of B6 mouse (a,b) and BALB/c mouse (c,d). Tachyzoites were indicated by white arrows and neutrophils were indicated by red arrows. Scale bar: a and d were $2 \mu \mathrm{m}$; b and c were $5 \mu \mathrm{m}$.

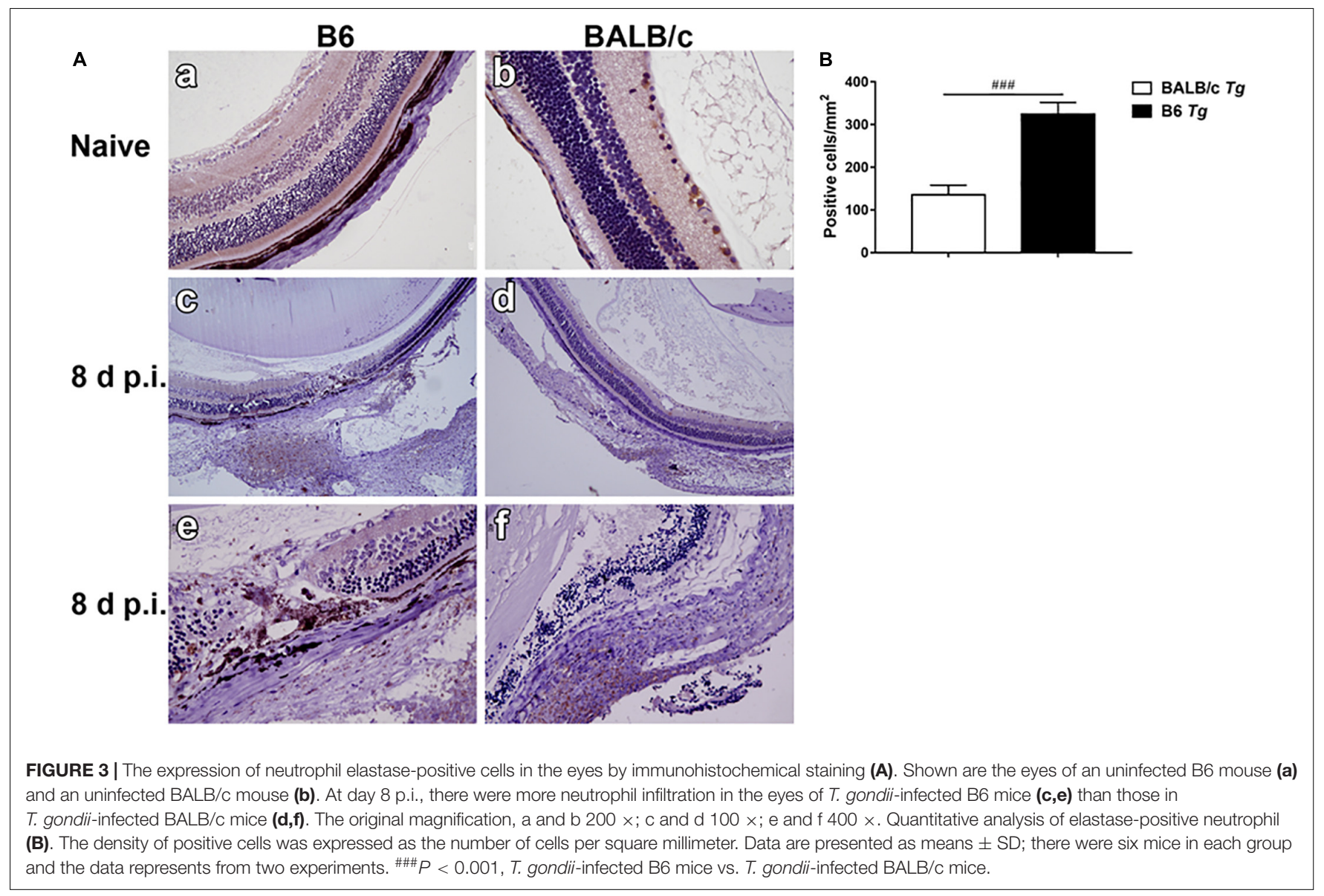




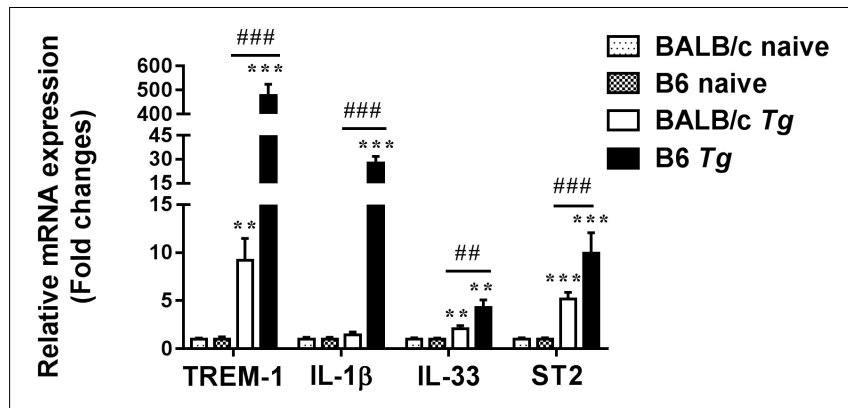

FIGURE 4 | The mRNA expressions of TREM-1, IL-1 $\beta$, IL-33, ST2, and TREM-2 in the eyes of naive mice and T. gondii-infected mice were measured by using qRT-PCR. Data are presented as means $\pm \mathrm{SD}$; there were six mice in each group and the data represents from two experiments. ${ }^{* *} P<0.01$, and ${ }^{* * *} P<0.001, T$. gondii-infected mice vs. naive mice. ${ }^{\# \#} P<0.01$, \#\#\# $P<0.001, T$. gondii-infected B6 mice vs. T. gondii-infected BALB/c mice.
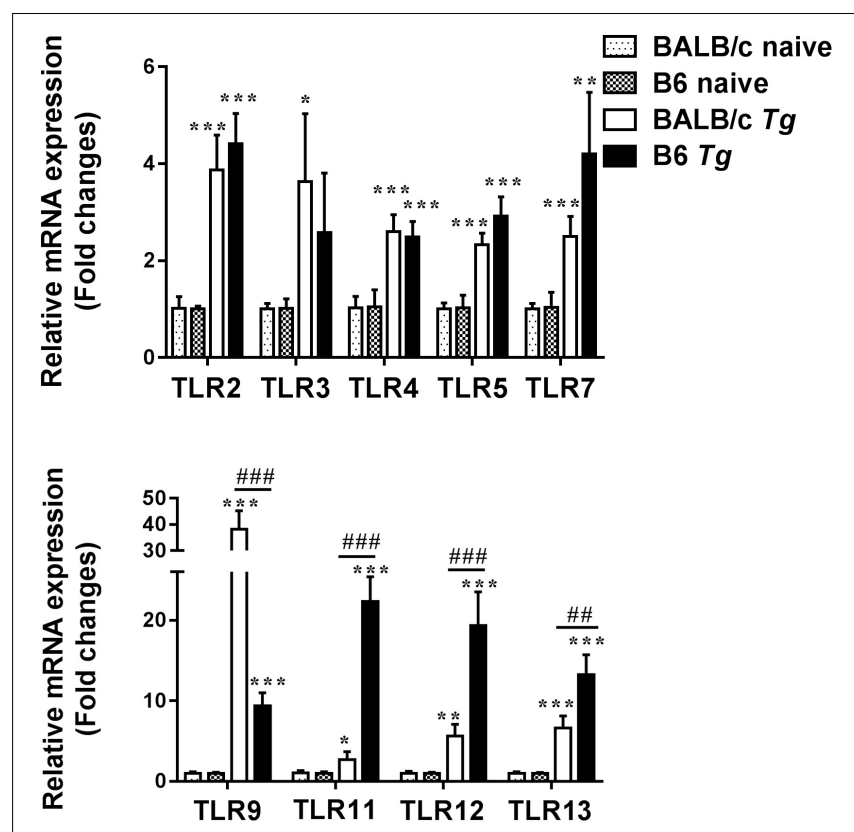

FIGURE 5 | The mRNA expressions of TLRs in the eyes of naive mice and T. gondii-infected mice were measured by using qRT-PCR. Data are presented as means $\pm S D$; there were six mice in each group and the data represents from two experiments. ${ }^{*} P<0.05,{ }^{*} P<0.01$, and ${ }^{* * *} P<0.001$, T. gondii-infected mice vs. naive mice. ${ }^{\#} P<0.01$ and $\# \# P<0.001$, T. gondii-infected B6 mice vs. T. gondii-infected BALB/c mice.

showed that there was greater neutrophil infiltration in the eyes of B6 mice than those in BALB/c mice following ocular T. gondii infection. The differences in expression levels of SAG1 gene and the numbers of neutrophils were consistent with the ocular pathological severity in the two strains of mice. Therefore, our concern is whether the effects of $T$. gondii infection on the expressions of TREM-1, TREM-2, IL-1 $\beta$, IL-33, ST2, and TLRs are differently in the eyes of T. gondii-infected B6 and $\mathrm{BALB} / \mathrm{c}$ mice.

Triggering receptor expressed on myeloid cells (TREM)-1, a cell surface receptor expressed at high levels on several immune cells such as polymorphonuclear neutrophils, macrophages, and monocytes, plays a vital role in innate and adaptive immune responses (Bouchon et al., 2000). TREM-1 activation increases the release of IL-6, TNF $\alpha$, and macrophage inflammatory protein-2 as well as polymorphonuclear neutrophil infiltration (Lagler et al., 2009); while TREM-2 may function as a negative regulator in the inflammatory response (Sun et al., 2013). So far, the expression of TREMs is an interesting and as yet unexplored player in OT. In this study, neutrophil infiltration and the expression levels of TREM-1, IL-33, and ST2 increased in the eyes of both $\mathrm{B} 6$ and $\mathrm{BALB} / \mathrm{c}$ mice, and the IL-1 $\beta$ level was increased in B6 mice after ocular T. gondii infection, TREM-1 plays a role in regulating neutrophil chemotaxis in acute infectious diseases and is a potential biomarker for the diagnosis of infectious diseases (Cao et al., 2017). TREM-1 alters neutrophil infiltration by stimulating AKT activation and NADPH oxidase-2-dependent superoxide release (Baruah et al., 2019). Blockage of TREM-1 expressed on neutrophils and monocytes/macrophages decreases the activation of neutrophils and monocytes/macrophages and mRNA expressions of inflammation-associated genes in alcoholic liver disease of mouse model (Tornai et al., 2019). IL-1 $\beta$ may play an important role in regulating the host's immune defense against T. gondii infection (Chang et al., 1990). It has been reported that IL-33 is released by damaged or necrotic cells, leading to activation of the immune system by ST2/IL-33 signaling (Moussion et al., 2008; Liew et al., 2010). ST2 increases the Th2 reaction and resistance to $P$. aeruginosa keratitis (Huang et al., 2007). Our previous study found that IL-33/ST2 axis may involve in the regulation of immunopathology of OT in Kunming mice (Tong and $\mathrm{Lu}, 2015$ ). In the present study, our data demonstrated that both TREM-1 and neutrophil infiltration are essential to T. gondii-stimulated ocular inflammatory response, and there were significant positive correlation between the mRNA expressions of TREM-1 and proinflammatory cytokine (IL-1 $\beta$ ) in the eyes of B6 mice, and between TREM-1 and IL33/ST2 in the eyes of both T. gondii-infected B6 and BALB/C mice. However, there were significantly higher numbers of neutrophil infiltration and higher levels of TREM-1, IL-1 $\beta$, and IL-33/ST2 in the eyes of susceptible B6 mice after ocular T. gondii infection, which indicate that upregulated TREM1 may promote inflammation through increased neutrophil recruitment and inflammatory cytokine production in the eyes of susceptible B6 mice.

Multiple TLRs contribute to host innate immunity to T. gondii infection, and different TLRs can induce distinctive immune responses to T. gondii (Sturge et al., 2013; Han et al., 2014). Our data showed that the expression levels of TLRs (including TLR2, TLR4, TLR5, TLR7, TLR9, TLR11, TLR12, and TLR13) were significantly elevated in the eyes of both $\mathrm{B} 6$ and $\mathrm{BALB} / \mathrm{c}$ mice infected with $T$. gondii through the ocular route. Besides, the levels of TLR11, TLR12, and TLR13 were significantly higher in susceptible B6 mice than those in resistant BALB/c mice, while TLR9 level was significantly higher in resistant BALB/c mice than that in B6 mice. During acute infection, T. gondii induces a protective immunity that is mainly Th1 cellular immune response (Denkers and Gazzinelli, 1998). After oral T. gondii infection, TLR-9 is crucial for an effective Th1-type 


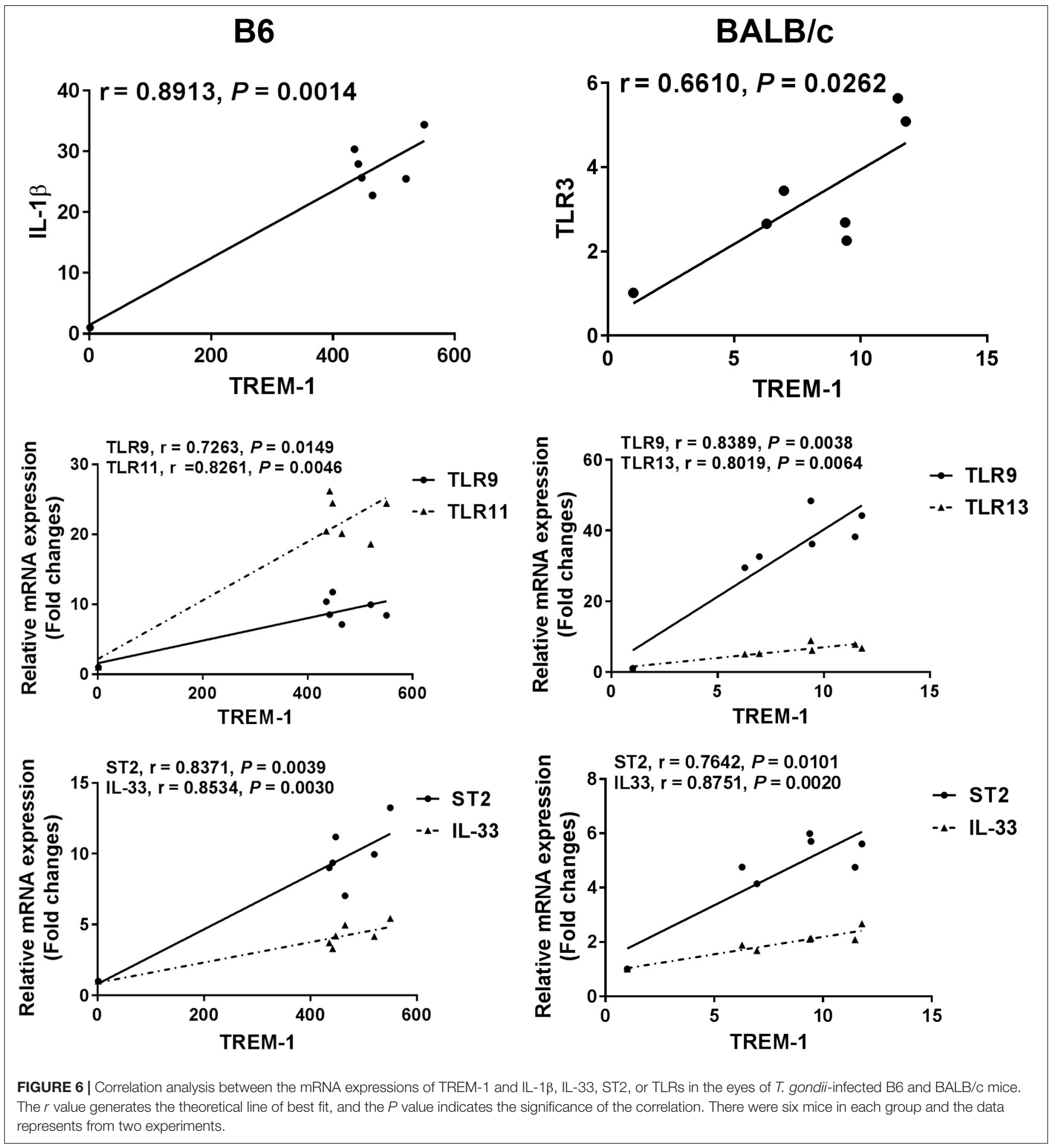

immune response in mice (Minns et al., 2006). T. gondii profilin recognized by TLR11/12 can induce an inflammatory response, and it can also induce innate and adaptive immune responses (Hedhli et al., 2016). In the current study, we observed that the pathological role of TREM-1 in OT may be associated with the expressions of TLR9/TLR11 in T. gondii-infected B6 mice, and associated with the expressions of TLR3/TLR9/TLR13 in
T. gondii-infected BALB/c mice, suggesting a possibly correlation between TREM-1 and TLR genes during acute OT. It has been reported that after infection with $P$. aeruginosa, TREM1 mRNA expressions were significantly increased in both human and mouse corneas, which contribute to amplifying corneal inflammation in $P$. aeruginosa keratitis by regulating TLR signaling and immune responses (Wu et al., 2011). 
Our data suggest that TLR3, TLR9, TLR11, TLR12, and TLR13 may play different roles during ocular $T$. gondii infection.

\section{CONCLUSION}

This study has provided evidences that following ocular T. gondii infection, increased neutrophil infiltration was consistent with the increase of TREM-1 expression in the eyes of both T. gondiiinfected $\mathrm{B} 6$ and $\mathrm{BALB} / \mathrm{C}$ mice, and significant correlations existed between TREM-1 and IL-1 $\beta /$ TLR9/TLR11/IL-33/ST2 in the eyes of B6 mice and existed between TREM-1 and TLR3/TLR9/TLR13/IL-33/ST2 in the eyes of BALB/c mice, which are possibly related to the ocular immunopathology. However, the functions of TREM-1 and the aforementioned genes related to $T$. gondii-induced immune and inflammatory responses in OT on different background of mice need to be further investigated.

\section{DATA AVAILABILITY STATEMENT}

The raw data supporting the conclusions of this manuscript will be made available by the authors, without undue reservation, to any qualified researcher.

\section{REFERENCES}

Ali-Heydari, S., Keshavarz, H., Shojaee, S., and Mohebali, M. (2013). Diagnosis of antigenic markers of acute toxoplasmosis by IgG avidity immunoblotting. Parasite 20:18. doi: 10.1051/parasite/2013017

Andrade, W. A., Souza, M. D. C., Ramos-Martinez, E., Nagpal, K., Dutra, M. S., Melo, M. B., et al. (2013). Combined action of nucleic acid-sensing Toll-like receptors and TLR11/TLR12 heterodimers imparts resistance to Toxoplasma gondii in mice. Cell Host Microbe. 13, 42-53. doi: 10.1016/j.chom.2012.12.003

Atmaca, L. S., Simsek, T., and Batioglu, F. (2004). Clinical features and prognosis in ocular toxoplasmosis. Jpn. J. Ophthalmol. 48, 386-391. doi: 10.1007/s10384003-0069-0

Baruah, S., Murthy, S., Keck, K., Galvan, I., Prichard, A., Allen, L. H., et al. (2019). TREM-1 regulates neutrophil chemotaxis by promoting NOX-dependent superoxide production. J. Leukoc. Biol. 105, 1195-1207. doi: 10.1002/JLB. 3VMA0918-375R

Beiting, D. P., Peixoto, L., Akopyants, N. S., Beverley, S. M., Wherry, E. J., Christian, D. A., et al. (2014). Differential induction of TLR3-dependent innate immune signaling by closely related parasite species. PLoS One 9:e88398. doi: 10.1371/ journal.pone.0088398

Bleharski, J. R., Kiessler, V., Buonsanti, C., Sieling, P. A., Stenger, S., Colonna, M., et al. (2003). A role for triggering receptor expressed on myeloid cells- 1 in host defense during the early-induced and adaptive phases of the immune response. J. Immunol. 170, 3812-3818. doi: 10.4049/jimmunol.170.7.3812

Bouchon, A., Dietrich, J., and Colonna, M. (2000). Cutting edge: inflammatory responses can be triggered by TREM-1, a novel receptor expressed on neutrophils and monocytes. J. Immunol. 164, 4991-4995. doi: 10.4049/ jimmunol.164.10.4991

Bouchon, A., Facchetti, F., Weigand, M. A., and Colonna, M. (2001). TREM-1 amplifies inflammation and is a crucial mediator of septic shock. Nature 410, 1103-1107. doi: 10.1038/35074114

Cao, C., Gu, J., and Zhang, J. (2017). Soluble triggering receptor expressed on myeloid cell-1 (sTREM-1): a potential biomarker for the diagnosis of infectious diseases. Front. Med. 11:169-177. doi: 10.1007/s11684-017-0505-Z

Carriere, V., Roussel, L., Ortega, N., Lacorre, D. A., Americh, L., Aguilar, L., et al. (2007). IL-33, the IL-1-like cytokine ligand for ST2 receptor, is a

\section{ETHICS STATEMENT}

Animal studies were conducted according to protocols approved by the Animal Experimentation Ethics Committee of Zhongshan School of Medicine on Laboratory Animal Care at Sun Yat-sen University (No. 2016-081), China.

\section{AUTHOR CONTRIBUTIONS}

FL designed the experiments, analyzed the data, wrote, and edited the manuscript. YZ conducted the experiments and analyzed the data. $\mathrm{JH}$ and $\mathrm{HZ}$ conducted the experiments. $\mathrm{SH}$ analyzed the data, revised, and edited the manuscript.

\section{FUNDING}

This work was supported by the Natural Science Foundation of China (No. 81971955); the Undergraduate Teaching Reform Project of Zhongshan School of Medicine in 2018, Sun Yat-sen University, China; the Key Development Project of Graduate School in 2019 (No. cxjh201908), Sun Yat-sen University, China.

chromatin-associated nuclear factor in vivo. Proc. Natl. Acad. Sci. U.S.A. 104, 282-287. doi: 10.1073/pnas.0606854104

Chang, H. R., Grau, G. E., and Pechere, J. C. (1990). Role of TNF and IL-1 in infections with Toxoplasma gondii. Immunology 69, 33-37.

Charles, E., Callegan, M. C., and Blader, I. J. (2007). The SAG1 Toxoplasma gondii surface protein is not required for acute ocular toxoplasmosis in mice. Infect. Immun. 75, 2079-2083. doi: 10.1128/iai.01685-06

Chen, S. J., Zhang, Y. X., Huang, S. G., and Lu, F. L. (2017). Galectins expressed differently in genetically susceptible $\mathrm{C} 57 \mathrm{BL} / 6$ and resistant $\mathrm{BALB} / \mathrm{c}$ mice during acute ocular Toxoplasma gondii infection. Parasitology 144, 1064-1072. doi: $10.1017 /$ S0031182017000270

Denkers, E. Y. (2010). Toll-like receptor initiated host defense against Toxoplasma gondii. J. Biomed. Biotechnol. 2010:737125. doi: 10.1155/2010/737125

Deckert-Schluter, M., Schluter, D., Schmidt, D., Schwendemann, G., Wiestler, O. D., and Hof, H. (1994). Toxoplasma encephalitis in congenic B10 and BALB mice: impact of genetic factors on the immune response. Infect. Immun. 62, 221-228.

Denkers, E. Y., and Gazzinelli, R. T. (1998). Regulation and function of T-cellmediated immunity during Toxoplasma gondii infection. Clin. Microbiol. Rev. 11, 569-588. doi: $10.1128 / \mathrm{cmr} .11 .4 .569$

Garweg, J. G. (2016). Ocular toxoplasmosis: an update. Klin. Monbl. Augenheilkd. 233, 534-539. doi: 10.1055/s-0041-111821

Griesenauer, B., and Paczesny, S. (2017). The ST2/IL-33 axis in immune cells during inflammatory diseases. Front. Immunol. 8:475. doi: 10.3389/fimmu.2017.00475

Han, S. J., Melichar, H. J., Coombes, J. L., Chan, S. W., Koshy, A. A., Boothroyd, J. C., et al. (2014). Internalization and TLR-dependent type I interferon production by monocytes in response to Toxoplasma gondii. Immunol. Cell. Biol. 92, 872-881. doi: 10.1038/icb.2014.70

Hatai, H., Lepelley, A., Zeng, W., Hayden, M. S., and Ghosh, S. (2016) Toll-Like receptor 11 (TLR11) interacts with flagellin and profilin through disparate mechanisms. PLoS One 11:e0148987. doi: 10.1371/journal.pone.014 8987

Hedhli, D., Moire, N., Akbar, H., Laurent, F., Heraut, B., Dimier-Poisson, I., et al. (2016). The antigen-specific response to Toxoplasma gondii profilin, a TLR11/12 ligand, depends on its intrinsic adjuvant properties. Med. Microbiol. Immunol. 205, 345-352. doi: 10.1007/s00430-016-0452-3 
Hu, L. T., Du, Z. D., Zhao, G. Q., Jiang, N., Lin, J., Wang, Q., et al. (2014). Role of TREM-1 in response to Aspergillus fumigatus infection in corneal epithelial cells. Int. Immunopharmacol. 23, 288-293. doi: 10.1016/j.intimp.2014.09.011

Huang, X., Du, W., Barrett, R. P., and Hazlett, L. D. (2007). ST2 is essential for Th2 responsiveness and resistance to Pseudomonas aeruginosa keratitis. Invest. Ophthalmol. Vis. Sci. 48, 4626-4633.

Jasper, S., Vedula, S. S., John, S. S., Horo, S., Sepah, Y. J., and Nguyen, Q. D. (2017). Corticosteroids as adjuvant therapy for ocular toxoplasmosis. Cochrane Database Syst. Rev. 1:CD007417. doi: 10.1002/14651858.CD007417.pub3

Jones, L. A., Roberts, F., Nickdel, M. B., Brombacher, F., McKenzie, A. N., Henriquez, F. L., et al. (2010). IL-33 receptor (T1/ST2) signalling is necessary to prevent the development of encephalitis in mice infected with Toxoplasma gondii. Eur. J. Immunol. 40, 426-436. doi: 10.1002/eji.200939705

Klesney-Tait, J., Turnbull, I. R., and Colonna, M. (2006). The TREM receptor family and signal integration. Nat. Immunol. 7, 1266-1273. doi: 10.1038/ni1411

Koblansky, A. A., Jankovic, D., Oh, H., Hieny, S., Sungnak, W., Mathur, R., et al. (2013). Recognition of profilin by Toll-like receptor 12 is critical for host resistance to Toxoplasma gondii. Immunity 38, 119-130. doi: 10.1016/j.immuni. 2012.09.016

Laboudi, M., and Sadak, A. (2017). Serodiagnosis of toxoplasmosis: the effect of measurement of IgG avidity in pregnant women in Rabat in Morocco. Acta Trop. 172, 139-142. doi: 10.1016/j.actatropica.2017.04.008

Lagler, H., Sharif, O., Haslinger, I., Matt, U., Stich, K., Furtner, T., et al. (2009). TREM-1 activation alters the dynamics of pulmonary IRAK-M expression in vivo and improves host defense during pneumococcal pneumonia. J. Immunol. 183, 2027-2036. doi: 10.4049/jimmunol.0803862

Liew, F. Y., Girard, J. P., and Turnquist, H. R. (2016). Interleukin-33 in health and disease. Nat. Rev. Immunol. 16, 676-689. doi: 10.1038/nri.2016.95

Liew, F. Y., Pitman, N. I., and McInnes, I. B. (2010). Disease-associated functions of IL-33: the new kid in the IL-1 family. Nat. Rev. Immunol. 10, 103-110. doi: $10.1038 /$ nri2692

Liu, J., Huang, S., and Lu, F. (2018). Galectin-3 and Galectin-9 may differently regulate the expressions of microglial M1/M2 markers and $\mathrm{T}$ helper 1/Th2 cytokines in the brains of genetically susceptible C57BL/6 and resistant BALB/C mice following peroral infection with Toxoplasma gondii. Front. Immunol. 9:1648. doi: 10.3389/fimmu.2018.01648

Lu, F., Huang, S., Hu, M. S., and Kasper, L. H. (2005). Experimental ocular toxoplasmosis in genetically susceptible and resistant mice. Infect. Immun. 73, 5160-5165. doi: 10.1128/IAI.73.8.5160-5165.2005

Lu, F., Huang, S., and Kasper, L. H. (2003). Interleukin-10 and pathogenesis of murine ocular toxoplasmosis. Infect. Immun. 71, 7159-7163. doi: 10.1128/iai. 71.12.7159-7163.2003

Lu, F., Huang, S., and Kasper, L. H. (2004). CD4+ T cells in the pathogenesis of murine ocular toxoplasmosis. Infect. Immun. 72, 4966-4972. doi: 10.1128/IAI. 72.9.4966-4972.2004

Maenz, M., Schluter, D., Liesenfeld, O., Schares, G., Gross, U., and Pleyer, U. (2014). Ocular toxoplasmosis past, present and new aspects of an old disease. Prog. Retin. Eye Res. 39, 77-106. doi: 10.1016/j.preteyeres.2013.12.005

Minns, L. A., Menard, L. C., Foureau, D. M., Darche, S., Ronet, C., Mielcarz, D. W., et al. (2006). TLR9 is required for the gut-associated lymphoid tissue response following oral infection of Toxoplasma gondii. J. Immunol. 176, 7589-7597. doi: 10.4049/jimmunol.176.12.7589

Moussion, C., Ortega, N., and Girard, J. P. (2008). The IL-1-like cytokine IL33 is constitutively expressed in the nucleus of endothelial cells and epithelial cells in vivo: a novel 'alarmin'? PLoS One 3:e3331. doi: 10.1371/journal.pone. 0003331

Mukherjee, S., Karmakar, S., and Babu, S. P. (2016). TLR2 and TLR4 mediated host immune responses in major infectious diseases: a review. Braz. J. Infect. Dis. 20, 193-204. doi: 10.1016/j.bjid.2015.10.011

Netea, M. G., Azam, T., Ferwerda, G., Girardin, S. E., Kim, S. H., and Dinarello, C. A. (2006). Triggering receptor expressed on myeloid cells-1 (TREM-1) amplifies the signals induced by the NACHT-LRR (NLR) pattern recognition receptors. J. Leukoc. Biol. 80, 1454-1461. doi: 10.1189/jlb.1205758
Park, Y. H., and Nam, H. W. (2013). Clinical features and treatment of ocular toxoplasmosis. Korean J. Parasitol. 51, 393-399. doi: 10.3347/kjp.2013.51. 4.393

Peixoto-Rangel, A. L., Miller, E. N., Castellucci, L., Jamieson, S. E., Peixe, R. G., Elias Lde, S., et al. (2009). Candidate gene analysis of ocular toxoplasmosis in Brazil: evidence for a role for toll-like receptor 9 (TLR9). Mem. Inst. Oswaldo. Cruz. 104, 1187-1190. doi: 10.1590/s0074-02762009000800019

Schenk, M., Bouchon, A., Seibold, F., and Mueller, C. (2007). TREM-1-expressing intestinal macrophages crucially amplify chronic inflammation in experimental colitis and inflammatory bowel diseases. J. Clin. Invest. 117, 3097-3106. doi: 10.1172/JCI30602

Sturge, C. R., Benson, A., Raetz, M., Wilhelm, C. L., Mirpuri, J., Vitetta, E. S., et al. (2013). TLR-independent neutrophil-derived IFN-gamma is important for host resistance to intracellular pathogens. Proc. Natl. Acad. Sci. U.S.A. 110, 10711-10716. doi: 10.1073/pnas.1307868110

Sun, M., Zhu, M., Chen, K., Nie, X., Deng, Q., Hazlett, L. D., et al. (2013). TREM-2 promotes host resistance against Pseudomonas aeruginosa infection by suppressing corneal inflammation via a PI3K/Akt signaling pathway. Invest. Ophthalmol. Vis. Sci. 54, 3451-3462. doi: 10.1167/iovs.12-10938

Tong, X., and Lu, F. (2015). IL-33/ST2 involves the immunopathology of ocular toxoplasmosis in murine model. Parasitol. Res. 114, 1897-1905. doi: 10.1007/ s00436-015-4377-3

Tornai, D., Furi, I., Shen, Z. T., Sigalov, A. B., Coban, S., and Szabo, G. (2019). Inhibition of triggering receptor expressed on myeloid cells 1 ameliorates inflammation and macrophage and neutrophil Activation in alcoholic liver disease in mice. Hepatol. Commun. 3, 99-115. doi: 10.1002/hep4.1269

Turnbull, I. R., Gilfillan, S., Cella, M., Aoshi, T., Miller, M., Piccio, L., et al. (2006). Cutting edge: TREM-2 attenuates macrophage activation. J. Immunol. 177, 3520-3524. doi: 10.4049/jimmunol.177.6.3520

van der Kraan, P. M., Stoop, R., Meijers, T. H., Poole, A. R., and van den Berg, W. B. (2001). Expression of type X collagen in young and old C57Bl/6 and $\mathrm{Balb} / \mathrm{c}$ mice. Relation with articular cartilage degeneration. Osteoarthr. Cartil. 9, 92-100. doi: 10.1053/joca.2000.0364

Watarai, H., Sekine, E., Inoue, S., Nakagawa, R., Kaisho, T., and Taniguchi, M. (2008). PDC-TREM, a plasmacytoid dendritic cell-specific receptor, is responsible for augmented production of type I interferon. Proc. Natl. Acad. Sci. U.S.A. 105, 2993-2998. doi: 10.1073/pnas.0710351105

Wu, M., Peng, A., Sun, M., Deng, Q., Hazlett, L. D., Yuan, J., et al. (2011). TREM1 amplifies corneal inflammation after Pseudomonas aeruginosa infection by modulating Toll-Like receptor signaling and Th1/Th2-Type immune responses. Infect. Immun. 79, 2709-2716. doi: 10.1128/IAI.00144-11

Wujcicka, W., Gaj, Z., Wilczynski, J., and Nowakowska, D. (2015). Possible role of TLR4 and TLR9 SNPs in protection against congenital toxoplasmosis. Eur. J. Clin. Microbiol. Infect. Dis. 34, 2121-2129. doi: 10.1007/s10096-0152461-3

Zhang, Y., Liang, Q., Liu, Y., Pan, Z., Baudouin, C., Labbe, A., et al. (2018). Expression of cytokines in aqueous humor from fungal keratitis patients. $B M C$ Ophthalmol. 18:105. doi: 10.1186/s12886-018-0754-x

Zhao, W., Che, C., Liu, K., Zhang, J., Jiang, N., Yuan, K., et al. (2018). Fenretinide inhibits neutrophil recruitment and IL-1beta production in Aspergillus fumigatus keratitis. Cornea 37, 1579-1585. doi: 10.1097/ICO. 0000000000001756

Conflict of Interest: The authors declare that the research was conducted in the absence of any commercial or financial relationships that could be construed as a potential conflict of interest.

Copyright (c) 2019 Zhang, He, Zheng, Huang and Lu. This is an open-access article distributed under the terms of the Creative Commons Attribution License (CC BY). The use, distribution or reproduction in other forums is permitted, provided the original author(s) and the copyright owner(s) are credited and that the original publication in this journal is cited, in accordance with accepted academic practice. No use, distribution or reproduction is permitted which does not comply with these terms. 